\title{
Dicyanometallates as model extended frameworks
}

\section{SUPPLEMENTARY INFORMATION}

\author{
Joshua A. Hill, Amber L. Thompson, and Andrew L. Goodwin* \\ Department of Chemistry, University of Oxford, Inorganic Chemistry Laboratory, \\ South Parks Road, Oxford OX1 3QR, U.K. \\ *To whom correspondence should be addressed; E-mail: andrew.goodwin@chem.ox.ac.uk.
}

Submitted to J. Am. Chem. Soc. 


\section{Methods}

\section{Single-crystal X-ray diffraction}

Single-crystal X-ray diffraction data of starting materials [PPN]Au $(\mathrm{CN})_{2}(\mathrm{~S} 1)$ and $[\mathrm{PPN}] \mathrm{Ag}(\mathrm{CN})_{2}$ (S2) were collected using an Oxford Diffraction (Rigaku Oxford Diffraction) SuperNova diffractometer fitted with an Oxford Cryosystems Cryostream 700 Plus open-flow nitrogen cooling device. ${ }^{\mathrm{S} 1}$ CrysAlisPro was used for data collection and reduction. ${ }^{\mathrm{S} 2}$ The structures were solved $a b$ initio using SIR92. ${ }^{S 3}$ All structures were refined with full-matrix least-squares on $F^{2}$ using CRYSTALS. ${ }^{\mathrm{S}}$, S5 Hydrogen atoms were visible in the difference Fourier map and treated in the usual manner. ${ }^{S 6}$ Key crystallographic details can be found in Table S1. Full structural data have been submitted to the CCDC as numbers 1444142-1444143. These data can also be obtained free of charge from The Cambridge Crystallographic Data Centre via http://www.ccdc.cam.ac.uk/data_request/cif.

Coefficients of thermal expansion for $\left[\mathrm{NBu}_{4}\right]_{0.5} \mathrm{Cd}\left[\mathrm{Ag}(\mathrm{CN})_{2}\right]_{2.5} 2$ were calculated using the web tool PASCal. ${ }^{\text {S7 }}$ Unit cells were determined from single crystal data collected using an Oxford Diffraction (Rigaku Oxford Diffraction) SuperNova diffractometer fitted with an Oxford Cryosystems Cryostream 700 Plus open-flow nitrogen cooling device. CrysAlisPro was used for data collection and reduction. ${ }^{\mathrm{S} 2}$ No phase transition was observed across the temperature range studied.

\section{Synthesis}

The sample of $[\mathrm{PPN}]_{0.5} \mathrm{Cd}\left[\mathrm{Ag}(\mathrm{CN})_{2}\right]_{2.5}(\mathrm{EtOH})(3 \mathrm{c})$ used for powder diffraction and TGA was was prepared by diffusion in a Schott bottle. Cold solutions of [PPN]Ag(CN) $)_{2}(1.108 \mathrm{~g}$ in $25 \mathrm{~mL}$ ethanol) and $\mathrm{Cd}\left(\mathrm{NO}_{3}\right)_{2} \cdot 4 \mathrm{H}_{2} \mathrm{O}(184 \mathrm{mg}$ in $7 \mathrm{~mL}$ ethanol) were carefully layered with a buffer of $50 \mathrm{~mL}$ cold ethanol separating the layers. Crystals formed overnight. A portion of these crystals, with mother liquor, were set aside in a sealed sample tube. The remaining product was filtered and thoroughly washed with cold ethanol then left to air-dry overnight yielding $300 \mathrm{mg}$ product (Yield: 69.6\%).

The sample of [PPN]Mn[Au(CN) $\left.)_{2}\right]_{3}$ (5a) used for powder diffraction and TGA was was prepared by diffusion in a Schott bottle. Cold solutions of [PPN]Au(CN) $)_{2}(1.060 \mathrm{~g}$ in $33 \mathrm{~mL}$ ethanol) and $\mathrm{Mn}\left(\mathrm{NO}_{3}\right)_{2} \cdot 4 \mathrm{H}_{2} \mathrm{O}(134 \mathrm{mg}$ in $4 \mathrm{~mL}$ ethanol) were carefully layered with a buffer of $20 \mathrm{~mL}$ cold ethanol separating the layers. Crystals formed overnight. A portion of these crystals, with mother liquor, were set aside in a sealed sample tube. The remaining product was filtered and thoroughly washed with cold ethanol then left to air-dry overnight yielding $423 \mathrm{mg}$ product (70.4\% yield).

The sample of $[\mathrm{PPN}] \mathrm{Cd}\left[\mathrm{Au}(\mathrm{CN})_{2}\right]_{3}$ (5b) used for powder diffraction and TGA was was prepared by diffusion in a Schott bottle. A cold solution of [PPN]Au(CN) $)_{2}(1.000 \mathrm{~g}$ in $30 \mathrm{~mL}$ ethanol) was carefully layered on top of a solution of $\mathrm{Cd}\left(\mathrm{NO}_{3}\right)_{2} \cdot 4 \mathrm{H}_{2} \mathrm{O}(132 \mathrm{mg}$ in $4 \mathrm{~mL}$ ethanol). Crystals formed overnight. A portion of these crystals, with mother liquor, were set aside in a sealed sample tube. The remaining product was filtered and thoroughly washed with cold ethanol then left to air-dry overnight yielding $436 \mathrm{mg}$ product $(73.6 \%$ yield). 
Table S1: Crystallographic details of $[\mathrm{PPN}] \mathrm{Au}(\mathrm{CN})_{2}(\mathbf{S 1})$ and $[\mathrm{PPN}] \operatorname{Ag}(\mathrm{CN})_{2}(\mathbf{S 2})$.

\begin{tabular}{|c|c|c|}
\hline $\begin{array}{l}\text { Compound } \\
\text { Empirical formula }\end{array}$ & $\begin{array}{l}\text { S1 } \\
\text { Au1P2N3C38H30 }\end{array}$ & $\begin{array}{l}\text { S2 } \\
\text { Ag1P2N3C38H30 }\end{array}$ \\
\hline Formula weight $\left(\mathrm{g} \mathrm{mol}^{-1}\right)$ & 787.59 & 698.49 \\
\hline Crystal System & Triclinc & Triclinc \\
\hline Space Group & $P \overline{1}$ (No. 2) & $P \overline{1}$ (No. 2) \\
\hline$Z$ & 2 & 2 \\
\hline Radiation $(\AA)$ & $\mathrm{Cu} \mathrm{K}_{\alpha}, \lambda=1.54180$ & $\mathrm{Cu} \mathrm{K}_{\alpha}, \lambda=1.54180$ \\
\hline Crystal colour & Colourless & Colourless \\
\hline Crystal size (mm) & $0.02 \times 0.01 \times 0.15$ & $0.04 \times 0.01 \times 0.13$ \\
\hline Temperature (K) & $280.0(2)$ & $280.0(4)$ \\
\hline$a(\AA)$ & $9.7335(3)$ & $9.7325(10)$ \\
\hline$b(\AA)$ & $11.0381(5)$ & $10.9975(14)$ \\
\hline$c(\AA)$ & $17.0129(5)$ & $17.099(2)$ \\
\hline$\alpha\left(^{\circ}\right)$ & $73.058(3)$ & $73.500(11)$ \\
\hline$\beta\left({ }^{\circ}\right)$ & $81.926(2)$ & $82.138(9)$ \\
\hline$\gamma\left({ }^{\circ}\right)$ & $79.666(3)$ & $80.190(10)$ \\
\hline$V\left(\AA^{3}\right)$ & $1712.60(11)$ & $1721.5(4)$ \\
\hline$\mu\left(\mathrm{mm}^{-1}\right)$ & 9.167 & 5.81 \\
\hline Reflections $(I>2 \sigma(I))$ & 6734 & 7229 \\
\hline $\mathrm{R}\left(F_{\mathrm{o}}\right)$ & 0.0207 & 0.0333 \\
\hline $\mathrm{R}_{w}\left(F_{0}\right)$ & 0.0524 & 0.0803 \\
\hline GOF & 0.9995 & 0.7792 \\
\hline
\end{tabular}

\section{Powder X-ray diffraction}

Room temperature powder X-ray diffraction patterns were collected using a PANalytical Empyrean diffractometer with $\mathrm{Cu} \mathrm{K}_{\alpha 1}$ radiation. Variable divergence slits were used with a fixed sample illumination diameter of $10 \mathrm{~mm}$ on a rotating sample. The instrument was fitted with 0.04 rad soller slits and a PIXCel ${ }^{1 \mathrm{D}}$ detector.

\section{Thermogravimetric Analysis}

Thermogravimetric analysis was carried out using a Perkin Elmer TGA-7 Thermogravimetric Analyzer. Quantities of between $1.5 \mathrm{mg}$ and $5 \mathrm{mg}$ were used for TGA. Each sample was held at $40^{\circ} \mathrm{C}$ for $5 \mathrm{~min}$ and then ramped to $1000^{\circ} \mathrm{C}$ at $5^{\circ} \mathrm{C} \mathrm{min}^{-1}$. 
Table S2: Variable temperature until cell parameters of $\left[\mathrm{NBu}_{4}\right]_{0.5} \mathrm{Cd}^{\mathrm{II}}\left[\mathrm{Ag}(\mathrm{CN})_{2}\right]_{2.5}$ determined from single crystal diffraction.

\begin{tabular}{lllllll} 
Run & $\mathrm{T} / \mathrm{K}$ & $a / \AA$ & $b / \AA$ & $c / \AA$ & $\beta /{ }^{\circ}$ & $\mathrm{V} / \AA^{3}$ \\
\hline 1 & 300 & $16.4746(10)$ & $13.7363(7)$ & $18.1177(10)$ & $105.035(6)$ & $3959.7(4)$ \\
2 & 100 & $16.2218(5)$ & $13.9534(3)$ & $18.0142(5)$ & $106.186(3)$ & $3915.186(3)$ \\
3 & 150 & $16.2725(3)$ & $13.9069(2)$ & $18.0406(3)$ & $105.9628(17)$ & $3925.17(11)$ \\
4 & 200 & $16.3346(3)$ & $13.849(19)$ & $18.0701(3)$ & $105.691(17)$ & $3935.47(11)$ \\
5 & 250 & $16.4028(3)$ & $13.7921(2)$ & $18.0929(3)$ & $105.3864(19)$ & $3946.43(12)$ \\
6 & 300 & $16.4766(4)$ & $13.7317(3)$ & $18.1122(4)$ & $105.035(2)$ & $3957.61(15)$ \\
7 & 350 & $16.5585(9)$ & $13.6675(6)$ & $18.1352(9)$ & $104.682(6)$ & $3970.2(4)$ \\
8 & 400 & $16.6326(14)$ & $13.6086(9)$ & $18.1559(13)$ & $104.248(9)$ & $3983.1(5)$
\end{tabular}

\section{$2\left[\mathrm{NBu}_{4}\right]_{0.5} \mathrm{Cd}\left[\mathrm{Ag}(\mathrm{CN})_{2}\right]_{2.5}$}

\section{Topology decomposition}

$\left.\left[\mathrm{NBu}_{4}\right]_{0.5} \mathrm{CdAg}(\mathrm{CN})_{2}\right]_{2.5}$ has a unique uninodal 5-connected topology with vertex symbol 4.4.6.6.6.6.6.6.6.6(3) (shortened to $4^{2} 6^{8}$ ). This self-catenating topology, jah1, can be decomposed, by breaking one linker, into two interpenetrating cds nets (Fig. S1).

Alternatively the structure can be understood by reference to another net. Looking down the $c$ axis, it can be thought of as pairs of corrugated, interpenetrating $(6,3)$ nets stacked in an $A B \ldots$ manner. These layers are connected by perpendicular struts of linear $\mathrm{Cd}-\mathrm{NC}-\mathrm{Ag}-\mathrm{CN}-\mathrm{Cd}$ linkages. The shortest connection between interpenetrated $(6,3)$ nets is via three nodes. The additional connectivity compared to the pairs of $(6,3)$ net results in a self-catenated single network.

\section{VT Single Crystal Diffraction}

Coefficients of thermal expansion were calculated from single crystal diffraction data for $\left[\mathrm{NBu}_{4}\right]_{0.5} \mathrm{Cd}\left[\mathrm{Ag}(\mathrm{CN})_{2}\right]_{2.5}(\mathbf{2})$. The unit cells used to calculate these values can be found in Table S2. Over the range 100-400 K 2 shows linear thermal expansion with coefficients of thermal expansion along the principal axes of $\alpha_{\mathrm{X}_{1}}=-83.1(1.6) \mathrm{MK}^{-1}, \alpha_{\mathrm{X}_{2}}=4.7(1.8) \mathrm{MK}^{-1}$, and $\alpha_{\mathrm{X}_{2}}=138(5) \mathrm{MK}^{-1}$ (Fig. S2). In terms of the unit cell axes $\mathrm{X}_{1}=\mathbf{b}, \mathrm{X}_{2}=-0.504 \mathbf{b}+0.864 \mathbf{c}$, and $\mathrm{X}_{3}=0.846 \mathrm{~b}-0.534 \mathbf{c}$.

\section{Oxide analogue of compounds 3a-c, $[\mathrm{PPN}]_{0.5} \mathbf{B X}_{2.5} \cdot\{\mathrm{EtOH}\}$}

The topology of $[\mathrm{PPN}]_{0.5} \mathrm{BX}_{2.5} \cdot\{\mathrm{EtOH}\}$ corresponds to the 3-dimensional 2-periodic sphere packing $(4,4)$ la. ${ }^{S 8}$ The closest oxide analogue of compounds $\mathbf{3 a}-\mathbf{c}$ are the $n=2$ Ruddlesden-Popper phases. Removal of the NaCl layers between the $n=2$ perovskite blocks 


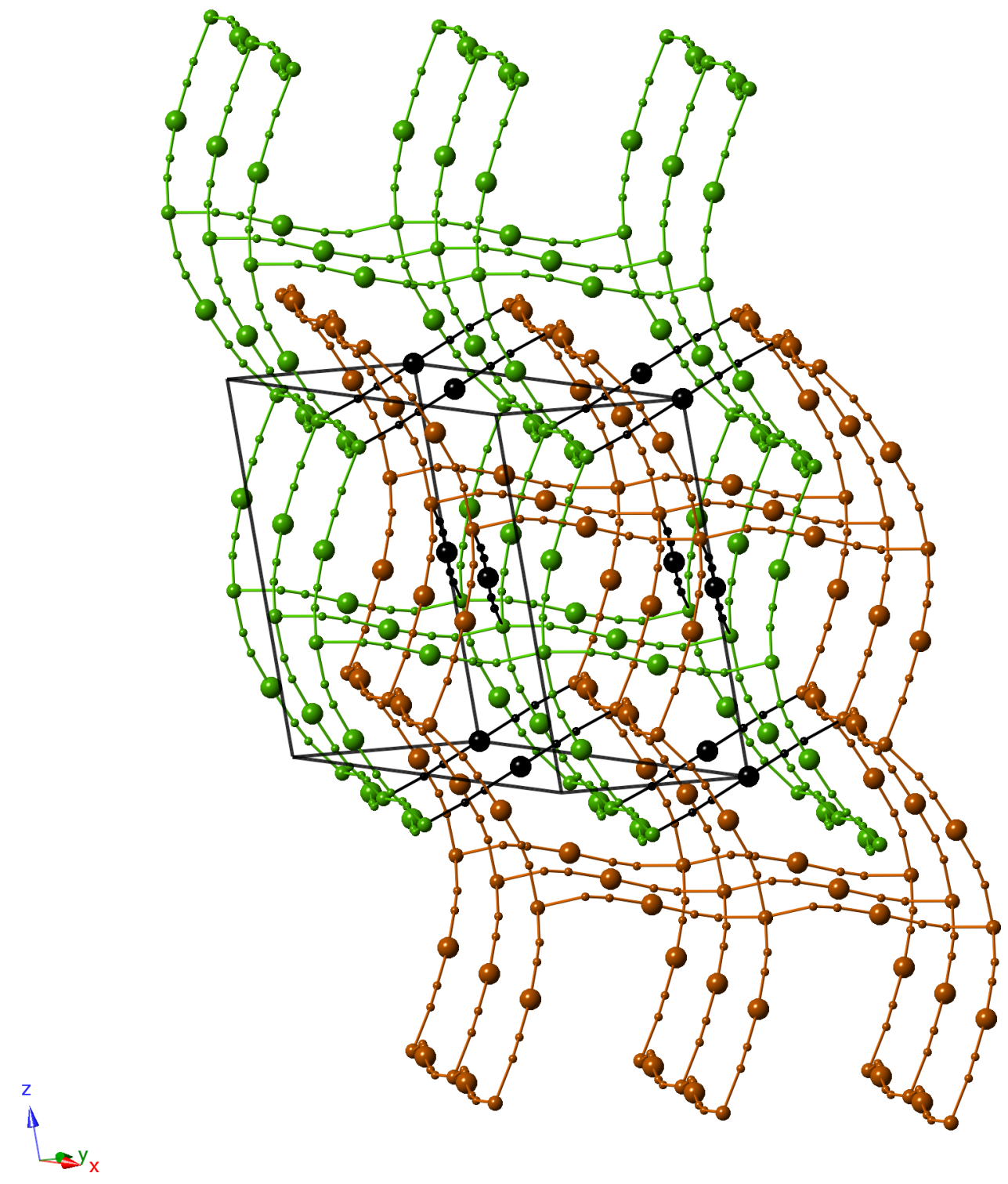

Figure S1: Decomposition of jah1 topology of $\left[\mathrm{NBu}_{4}\right]_{0.5} \mathrm{Cd}\left[\mathrm{Ag}(\mathrm{CN})_{2}\right]_{2.5}$. The topology can be decomposed into two interpenetrating cds nets (green and brown) by breaking the linkers shown in black.

in $\mathrm{A}_{3} \mathrm{~B}_{2} \mathrm{O}_{7}$ would leave behind $\mathrm{AB}_{2} \mathrm{O}_{5}$ layers with an $\mathrm{AB}$... stacking sequence just like compouds 3a-c. 


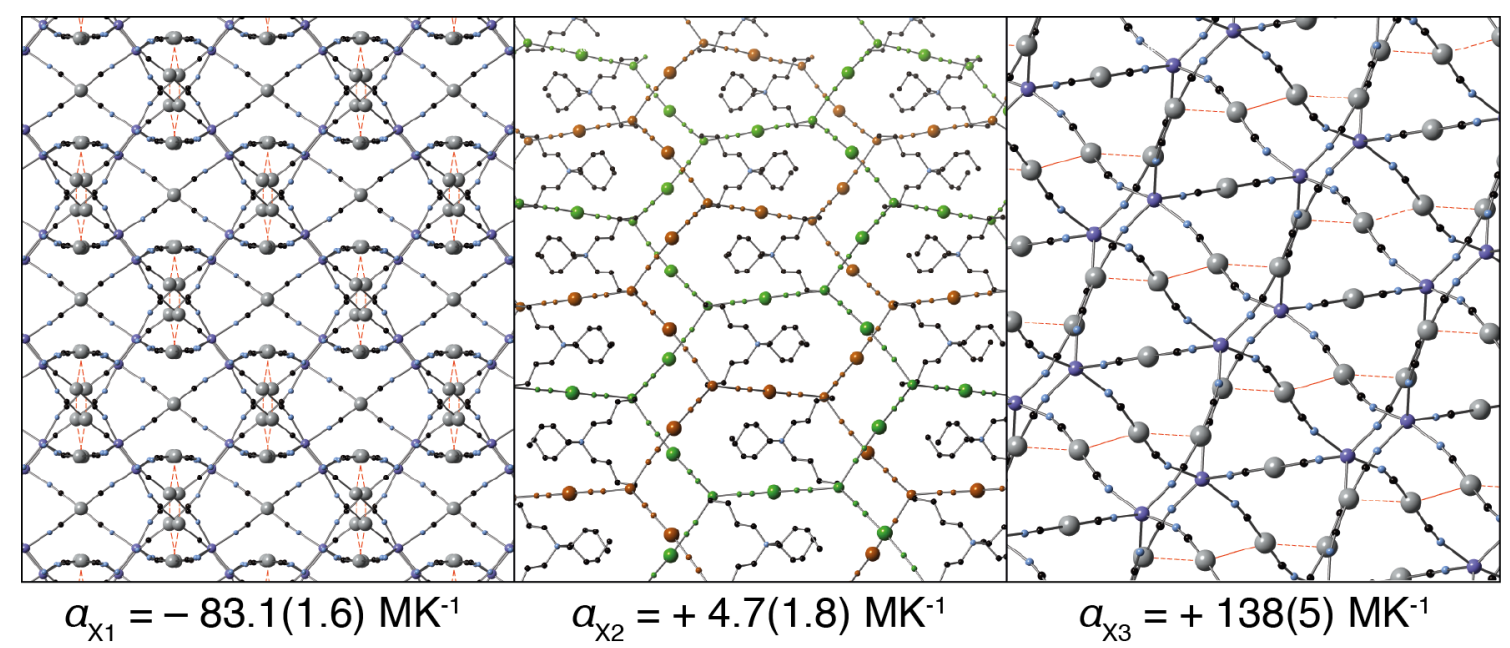

Principal axes

Figure S2: Views of the three principal axes of thermal expansion and their relationship to the crystal structure. Ag... Ag distances of less than $3.44 \AA$ are indicated by red dashed lines. Cadmium (purple), silver (grey), nitrogen (blue), carbon (black). In the central panel only a single pair of interwoven $(6,3)$ nets is shown (green and brown) and the tetra(n-butyl)ammonium extra-framework cation is shown.

\section{Tolerance factors of superperovskites}

Tolerance factors, $\alpha$ for various 'superperovskites' were calculated using a method adapted from Ref. 9. As detailed in that paper, Goldschmidt's tolerance factor (eqn. 1):

$$
\alpha=\left(\boldsymbol{r}_{\mathrm{A}}+\boldsymbol{r}_{\mathrm{X}}\right) / \sqrt{2}\left(\boldsymbol{r}_{\mathrm{B}}+\boldsymbol{r}_{\mathrm{X}}\right),
$$

where $\boldsymbol{r}_{\mathrm{i}}$ are the ionic radii for the species in a perovskite $A B X_{3}$, can be adapted for systems with molecular $A$ and $X$ species. Effective radii for $A$ and $X$ must be defined. This is done by measuring the radius from the centre of mass of the species to the outermost atom (from crystallographic data) and, to this number, adding the radius of the outermost atom. For example $\boldsymbol{r}_{\mathrm{A}_{\mathrm{eff}}}=\boldsymbol{r}_{\mathrm{A}_{\mathrm{mass}}}+\boldsymbol{r}_{\mathrm{A}_{\mathrm{ion}}}$. Combining this approach with Goldschmidt's we have:

$$
\alpha=\left(\boldsymbol{r}_{\mathrm{A}_{\mathrm{eff}}}+\boldsymbol{r}_{\mathrm{X}_{\mathrm{eff}}}\right) / \sqrt{2}\left(\boldsymbol{r}_{\mathrm{B}}+0.5 \boldsymbol{h}_{\mathrm{X}_{\mathrm{eff}}}\right),
$$

where $\boldsymbol{h}_{\mathrm{X}_{\mathrm{eff}}}$ is the length of the molecular linker. Values of $\boldsymbol{r}_{\mathrm{A}_{\text {mass }}}$ and $\boldsymbol{r}_{\mathrm{X}_{\text {mass }}}$ were measured from single crystal data and combined with tabulated data for ionic radii and covalent radii. $S 10, S 11$. Where there are crystallographical distinct $X$ both were measured and a mean taken. In the case of disordered crystal structures a mean position was used. Numerical values are listed in Table S3.

While these PPN systems lack hydrogen bonding it is important to treat these tolerance factors rather cautiously. Given the flexibility of dicyanometallate linkers, the variety of conformations known for [PPN $]^{+}$, and problems with defining appropriate sets of radii. However, 
Table S3: Tolerance factor calculations for some PPN superperovskites. COM stands for centre of mass. All distances in Å.

\begin{tabular}{|c|c|c|c|c|c|c|c|}
\hline Compound & $r_{\mathrm{A}_{\mathrm{eff}}}$ & $r_{\mathrm{X}_{\mathrm{eff}}}$ & $\boldsymbol{r}_{\mathrm{B}}$ & $\boldsymbol{h}_{\mathrm{X}}$ eff & $\alpha$ & A COM to edge & $\boldsymbol{r}$ (outer elem) \\
\hline $\mathrm{PPNMn}\left[\mathrm{Au}(\mathrm{CN})_{2}\right]_{3}(\mathbf{5 b})$ & 6.31 & 1.482 & 0.83 & 9.148 & 1.01957366 & 5.58 & 0.73 \\
\hline $\mathrm{PPNCd}\left[\mathrm{Au}(\mathrm{CN})_{2}\right]_{3}(\mathbf{5 c})$ & 6.3 & 1.467 & 0.95 & 9.111 & 0.99756577 & 5.57 & 0.73 \\
\hline Compound & $\mathrm{X}$ COM to central atom & central atom & $r$ (central atom) & $\mathrm{X}$ COM to end & $\boldsymbol{r}$ (end atom) & Temperature / K & \\
\hline $\operatorname{PPNMn}\left[\mathrm{Au}(\mathrm{CN})_{2}\right]_{3}(\mathbf{5 b})$ & 0.112 & $\mathrm{Au}$ & 1.37 & 3.114 & 1.46 & 150 & \\
\hline PPNNi[Au(CN $\left.)_{2}\right]_{3} \mathrm{~S} 13$ & 0.067 & $\mathrm{Au}$ & 1.37 & 3.1 & 1.46 & 293 & \\
\hline
\end{tabular}

they could be extremely useful for identifying the interactions that cause the tolerance factor concept to breakdown in these, and other, ${ }^{S 12}$ systems.

\section{Dicyanometallate tilt systems}

The tilt systems of compounds $\mathbf{5 a}$ and $\mathbf{5 a}$ both correspond to the conventional Glazer notation $a^{-} a^{-} c^{-}$. 14 However, compound $\mathbf{4}$ shows in-phase tilting of adjacent octahedra that propagates along a direction perpendicular to the rotation axis. Such a correlation is geometrically forbidden for perovskites such as oxides and halides, where neighbouring octahedra are connected by monatomic anions [Fig S3]. ${ }^{\mathrm{S} 14, \mathrm{~S} 15}$ In molecular perovskite analogues there will be many more possible tilt systems than can be described using Glazer notation (such as in 4). Consequently, we proceed to generalise the Glazer notation to allow succinct distinction of arbitrary tilt systems in these molecular systems.

Our starting point is to recall the relationship between tilt axis and propagation wave-vector in conventional perovskites. In principle, octahedral tilts around the a axis can propagate with wave-vector $\mathbf{k}=\left[k_{1}, \frac{1}{2}, \frac{1}{2}\right]$ : the sense of rotation strictly alternates within the (100) plane but rotations between neighbouring (100) planes need not be correlated. Tilts around $\mathbf{b}$ and $\mathbf{c}$ are similarly constrained to $\mathbf{k}=\left[\frac{1}{2}, k_{2}, \frac{1}{2}\right]$ and $\left[\frac{1}{2}, \frac{1}{2}, k_{3}\right]$ respectively; to first order these three tilt systems can operate independently. Taken together, this means that all physically realisable tilt systems of conventional perovskites can be represented by the three wave-vector components $k_{1}, k_{2}, k_{3}$ and the parameters $\epsilon_{1}, \epsilon_{2}, \epsilon_{3} \in\{0,1\}$ which represent whether a tilt axis is active $(\epsilon=1)$ or not $(\epsilon=0)$. Empirically, the overwhelming majority of tilt systems correspond to the special cases of "in-phase" $\left(k_{i}=0\right)$ and "out-of-phase" tilts $\left(k_{i}=\frac{1}{2}\right)$, although exceptions are not unknown. ${ }^{S 16}$ The "+/0/-" components of Glazer notation reflect these special cases:

$$
\begin{array}{rlrl}
+ & \epsilon_{i}=1 & k_{i}=0 \\
0 & \epsilon_{i} & =0 \\
-\quad \epsilon_{i} & =1 & & \\
& k_{i}=\frac{1}{2}
\end{array}
$$

Or, equivalently, the Glazer symbol $g_{i}$ is given by $\epsilon_{i} \exp \left[2 \pi \mathrm{i} k_{i}\right]$.

With respect to allowed tilt systems, the key conceptual difference between conventional perovskites and molecular perovskite analogues is that tilts can be correlated with arbitrary 


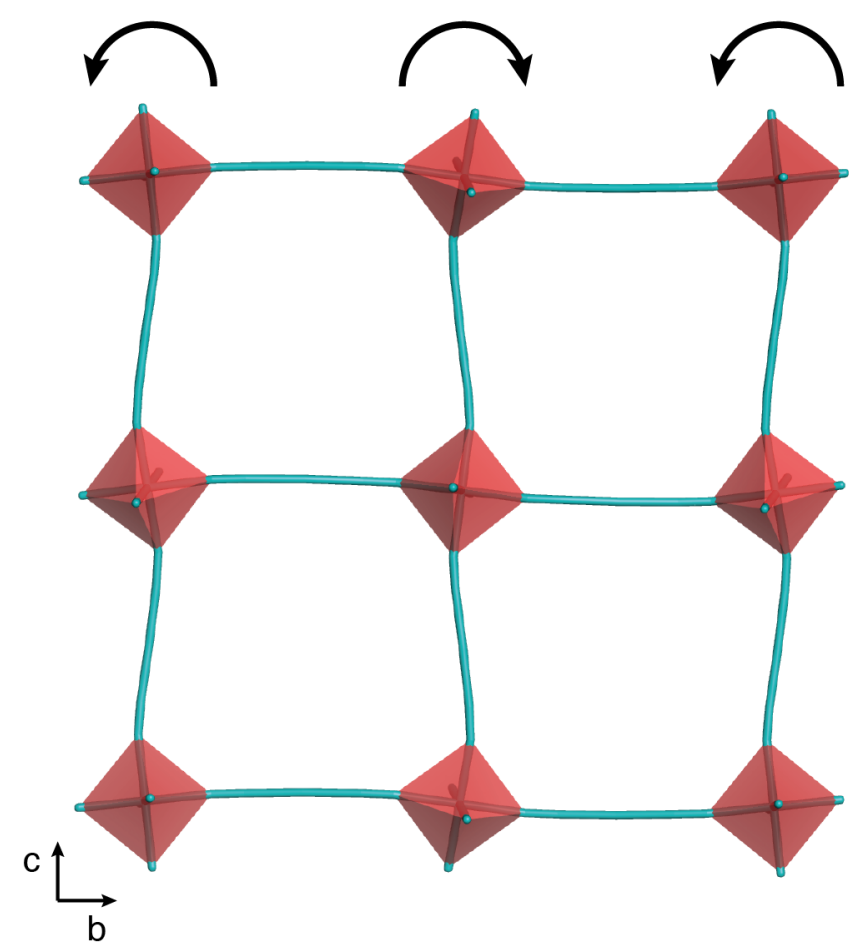

Figure S3: Compound 4 exhibits an octahedral tilt system for which there is no analogue in conventional perovskites (i.e., with octahedra connected by monoatomic anions). Here we consider the sense (clockwise/anticlockwise) of tilts about the [100] axis. For the left-hand column, all octahedra are tilted in an anticlockwise sense; for the central column the tilts are clockwise; and for the right-hand column the tilts are again anticlockwise. In conventional perovskites, the sense of octahedral rotation must alternate within the plane perpendicular to the tilt axis, so columns of octahedra cannot tilt in the same sense, as we show here.

wave-vector for the latter family. Consequently, any general notation to describe tilts in molecular perovskites must allow for three wave-vector components corresponding to each rotation axis. There are now nine Glazer terms

$$
g_{i j}=\epsilon_{i} \exp \left[2 \pi \mathrm{i} k_{i j}\right],
$$

describing the propagation of tilts about axis $i$ along axis $j$; here $\epsilon_{i}$ is as above, and $k_{i j}$ is the $j^{\text {th }}$ wave-vector component for the axis- $i$ tilt system. The $g_{i j}$ can be assembled into a single tensor $\mathbf{G}$, which we suggest is the most economical means of characterising these more complex tilt systems. 
For the specific example of compound $\mathbf{4}$, we have approximately

$$
\mathbf{G}=\left[\begin{array}{lll}
- & - & + \\
+ & - & - \\
- & + & -
\end{array}\right],
$$

where we use - to mean -1 and + to mean 1 . For clarity, the meaning of this tensor is that tilts around a propagate with $\mathbf{k}=\left[\frac{1}{2}, \frac{1}{2}, 0\right]$, tilts around $\mathbf{b}$ propagate with $\mathbf{k}=\left[0, \frac{1}{2}, \frac{1}{2}\right]$ and tilts around c propagate with $\mathbf{k}=\left[\frac{1}{2}, 0, \frac{1}{2}\right]$. We note that for conventionally-allowed tilt systems this notation reduces straightforwardly to conventional Glazer symbols: $g_{i}=g_{i i}$. 


\section{Powder diffraction}

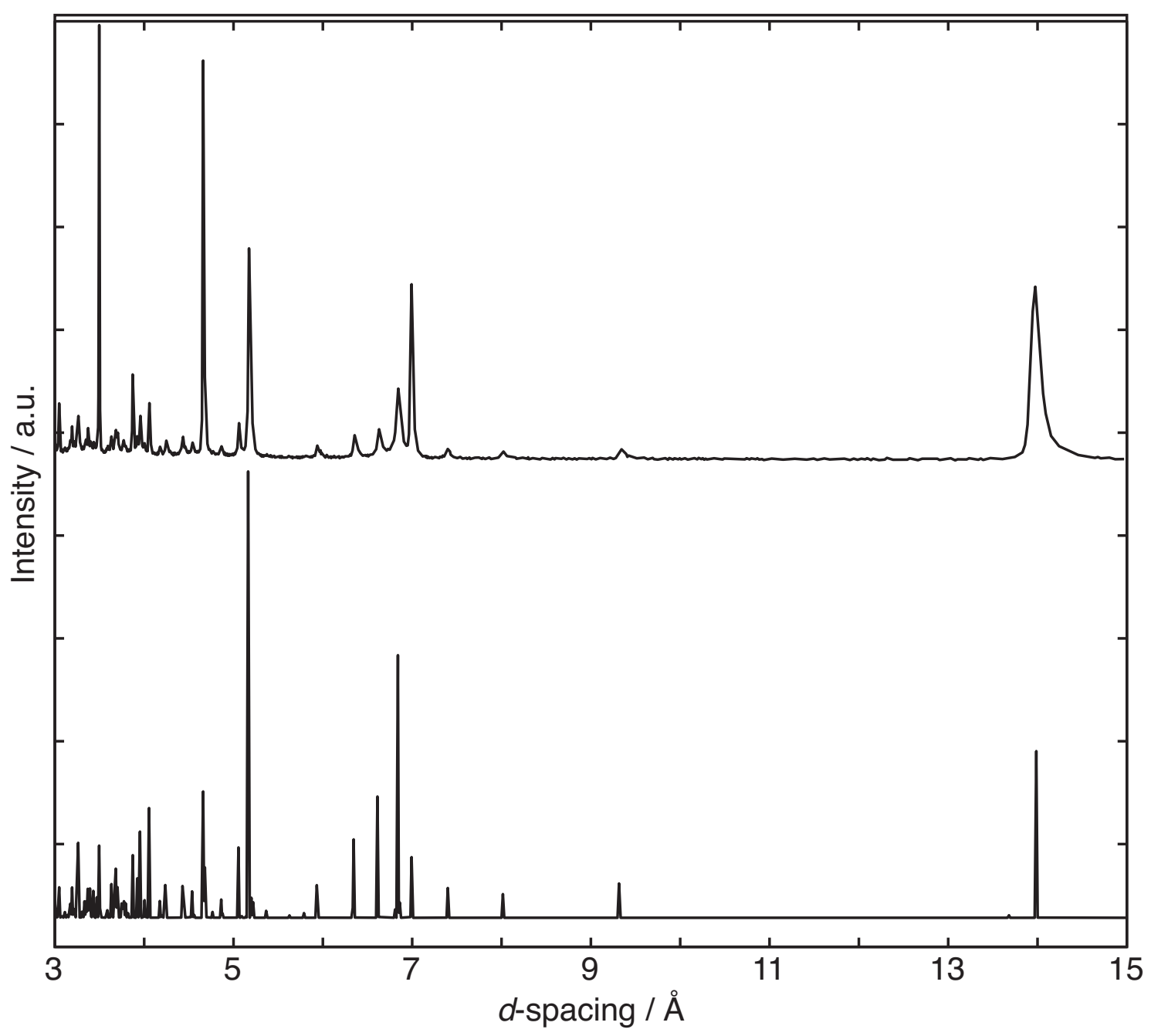

Figure S4: Experimental (top) and predicted (bottom) powder patterns for $\mathbf{3 b}$. The significant difference in intensity is due to preferred orientation, resulting in relatively strong $\{$ h00 $\}$ peaks. 


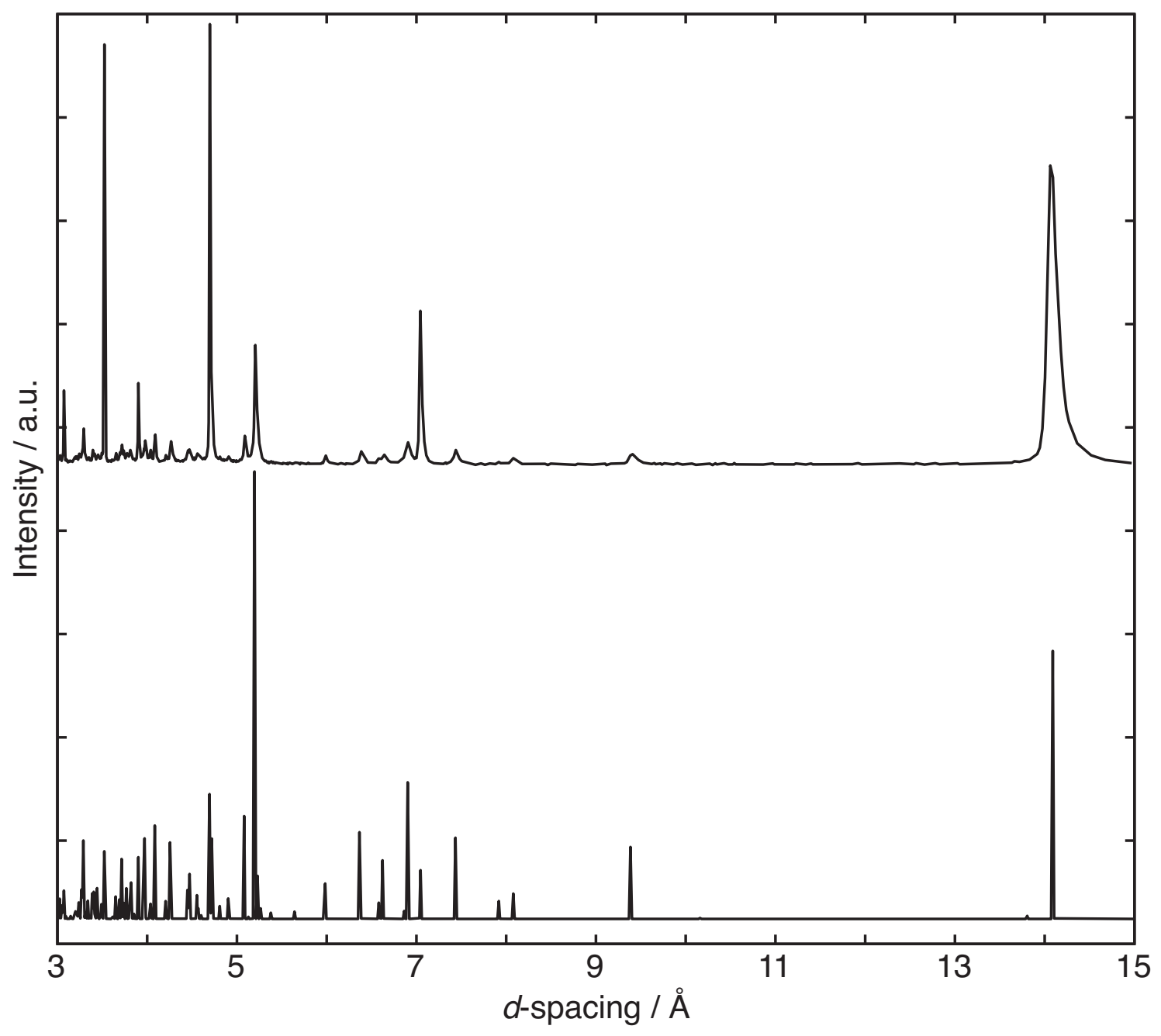

Figure S5: Experimental (top) and predicted (bottom) powder patterns for 3c. The significant difference in intensity is due to preferred orientation, resulting in relatively strong $\{$ h00 $\}$ peaks. 


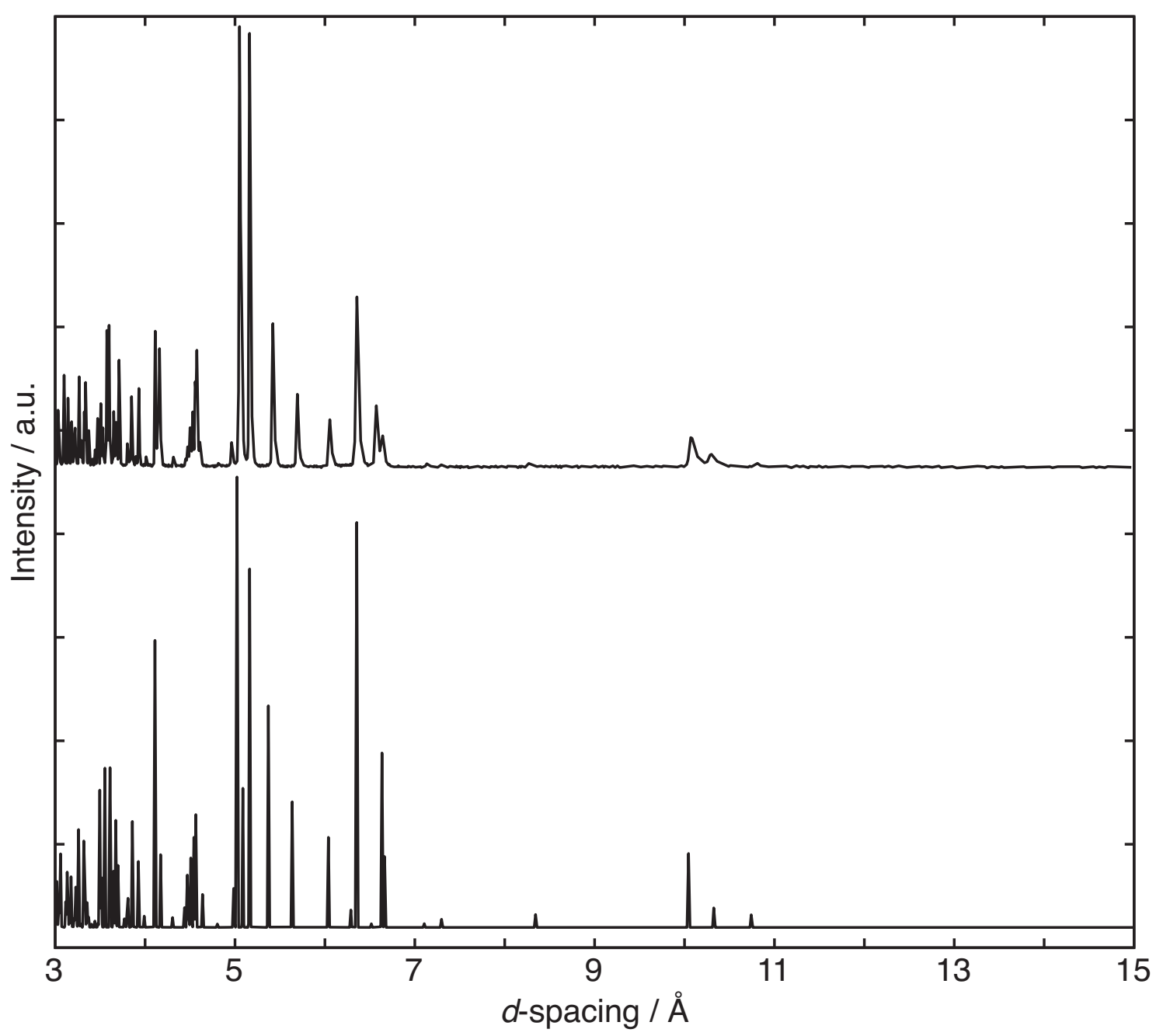

Figure S6: Experimental (top) and predicted (bottom) powder patterns for 5a. The powder data were collected at ambient temperature whereas the predicted pattern is derived from single-crystal data collected at $150 \mathrm{~K}$. 


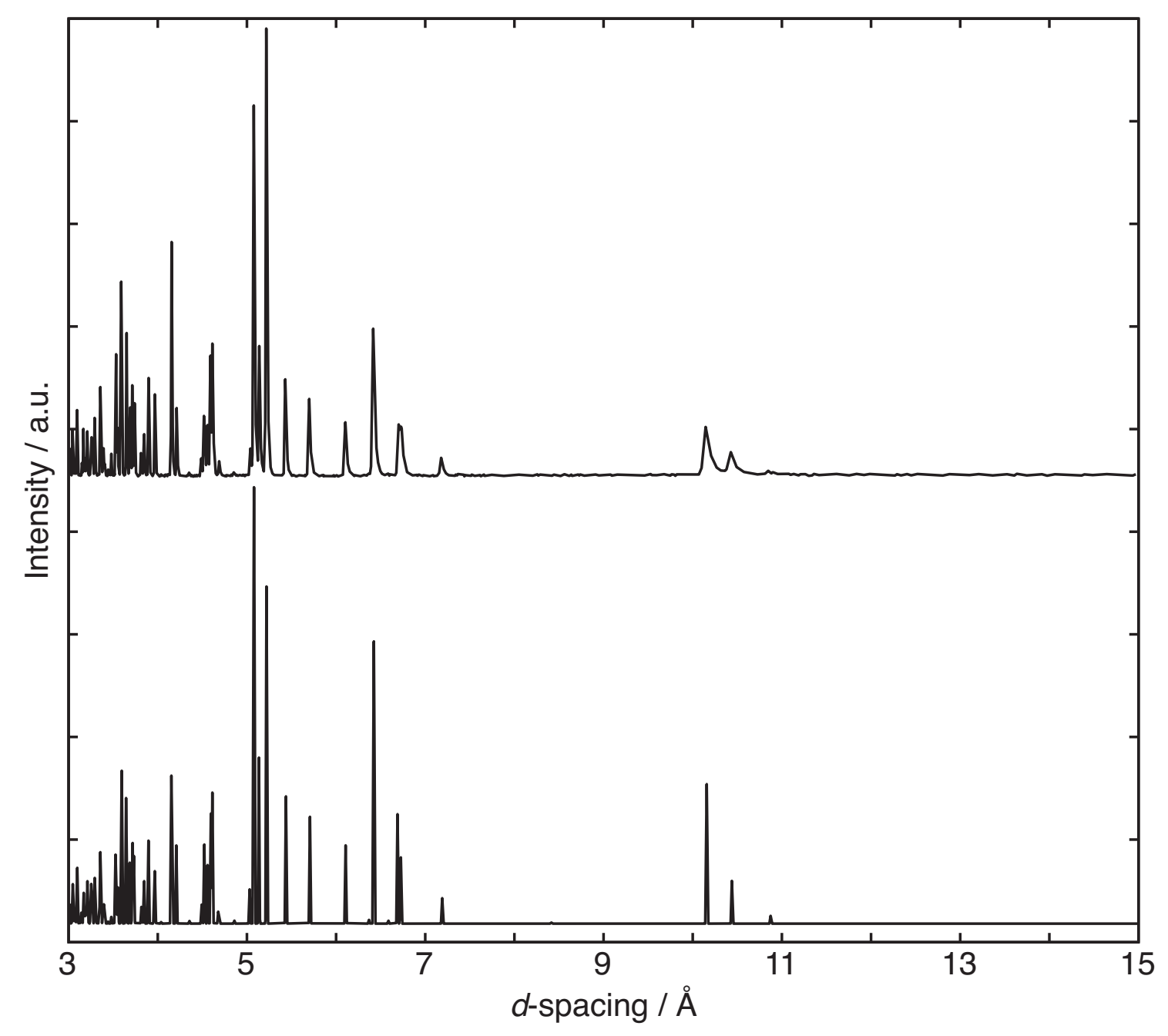

Figure S7: Experimental (top) and predicted (bottom) powder patterns for $\mathbf{5 b}$. 


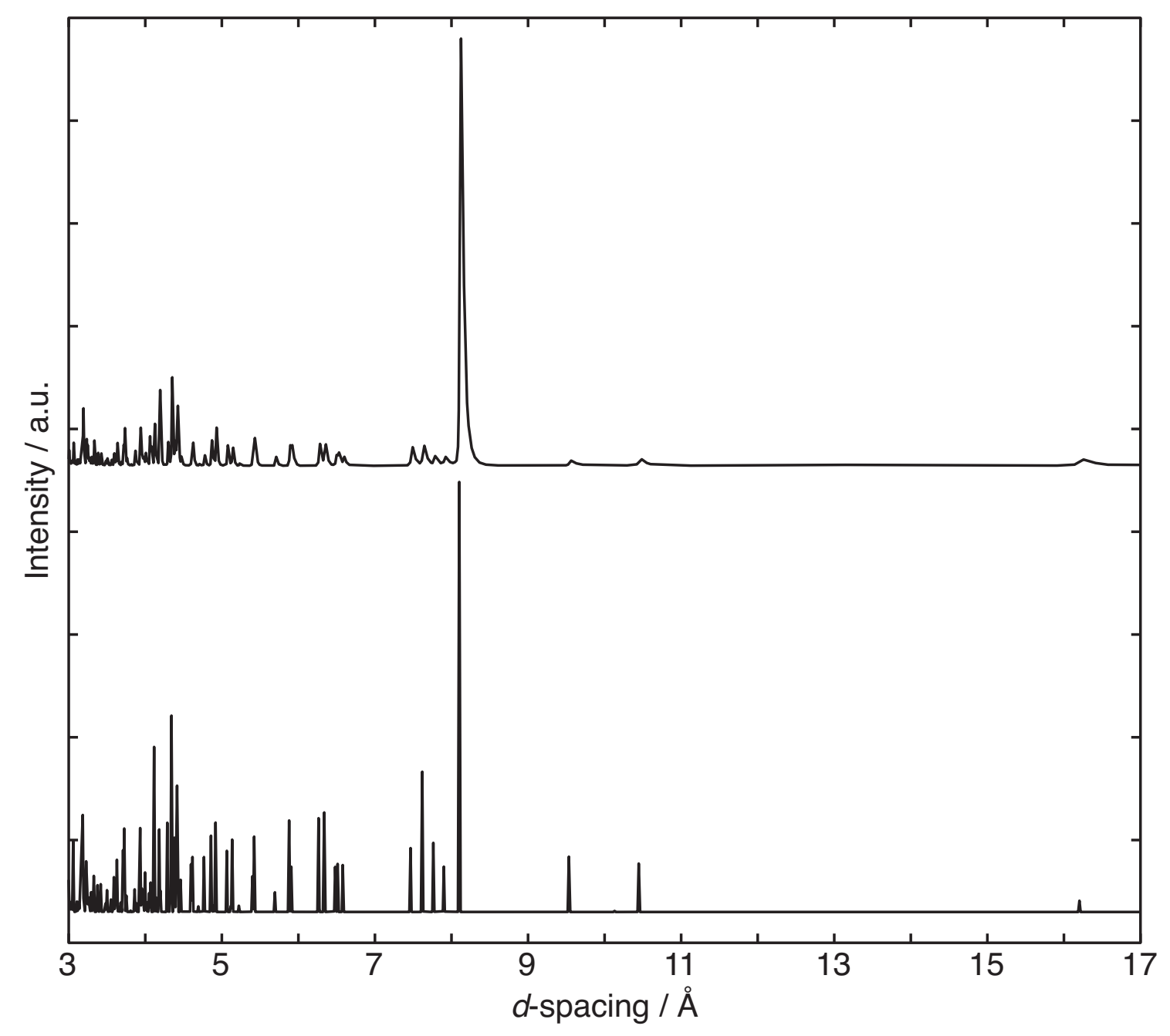

Figure S8: Experimental (top) and predicted (bottom) powder patterns for S1. 


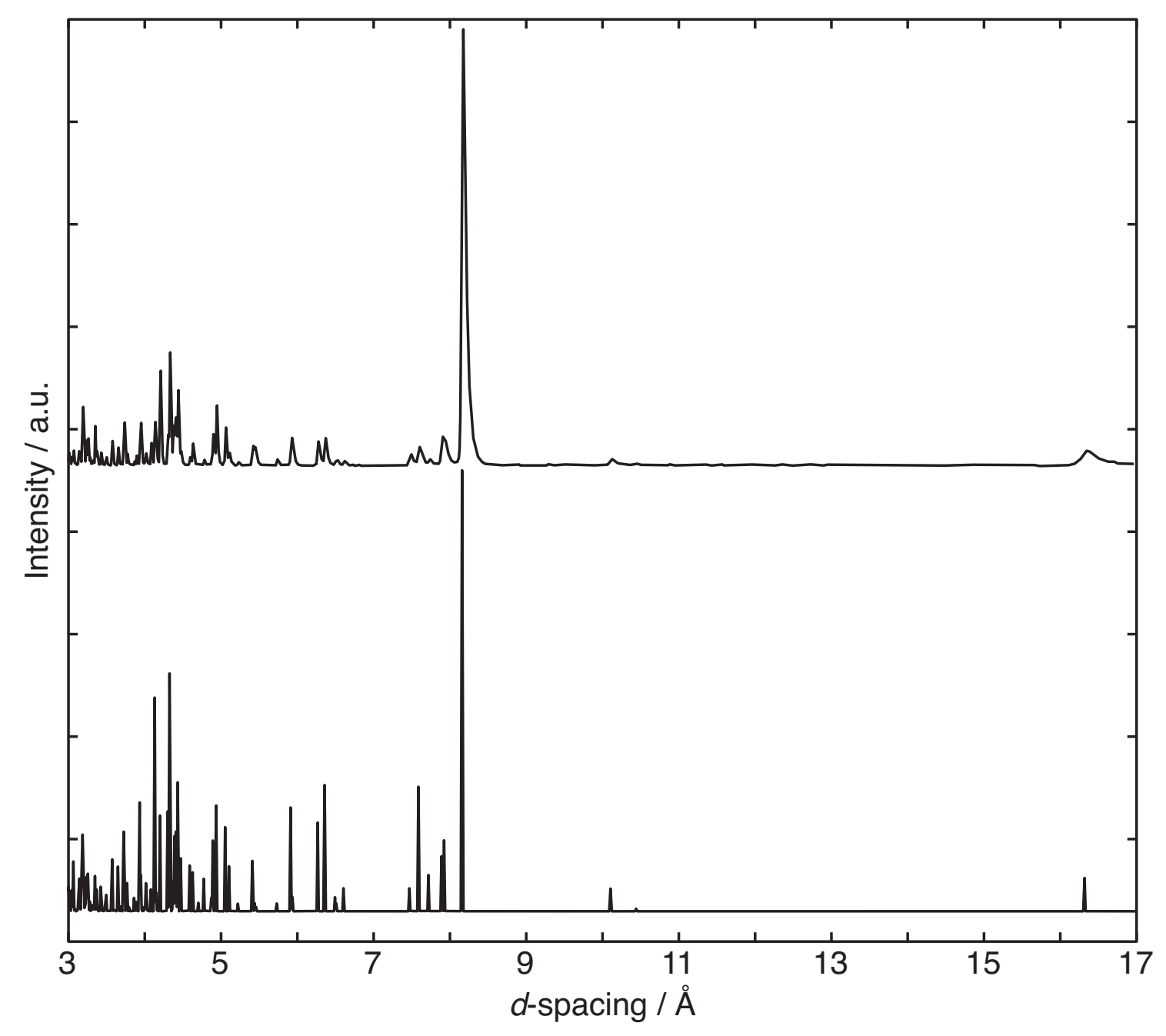

Figure S9: Experimental (top) and predicted (bottom) powder patterns for S2. 


\section{Thermogravimetric analysis}

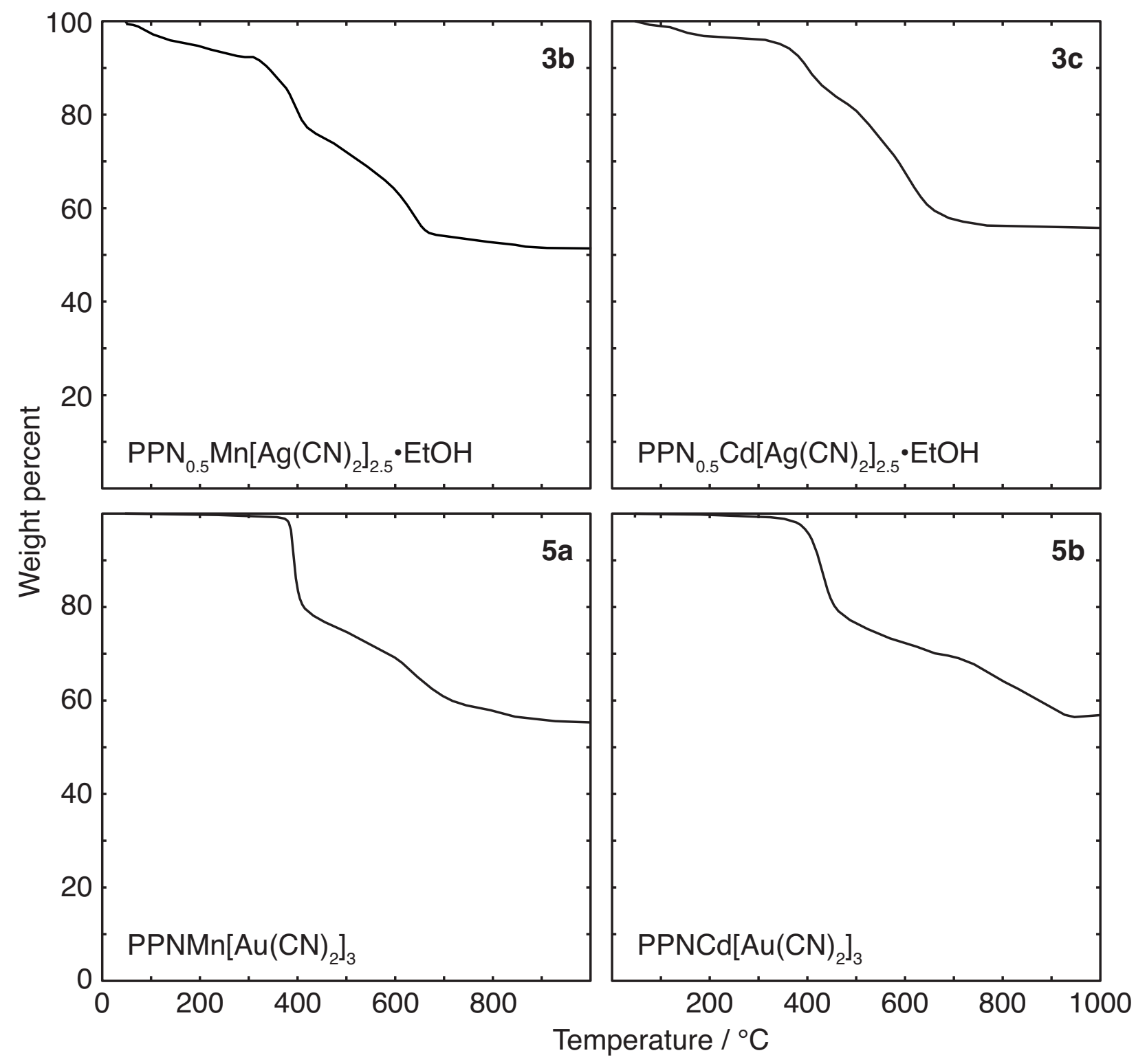

Figure S10: Thermogravimetric analysis for samples $\mathbf{3 b}, \mathbf{3 c}, \mathbf{5 a}$, and $\mathbf{5 c}$. Loss of ethanol prior to decomposition is evident for $\mathbf{3} \mathbf{b}$ and $\mathbf{3} \mathbf{c}$. 


\section{References}

(S1) Cosier, J.; Glazer, A. M. J. Appl. Crystallogr., 1986, 19, 105-107.

(S2) Crysalis PRO; Agilent Technologies: Yarnton, England, 2010.

(S3) Altomare, A.; Cascarano, G.; Giacovazzo, C.; Guagliardi, A.; Burla, M. C.; Polidori, G.; Camalli, M. J. Appl. Crystallogr., 1994, 27, 435-435.

(S4) Betteridge, P. W.; Carruthers, J. R.; Cooper, R. I.; Prout, K.; Watkin, D. J. J. Appl. Crystallogr., 2003, 36, 1487-1487.

(S5) Parois, P.; Cooper, R. I.; Thompson, A. L. Chem. Cent. J., 2015, 9, 30.

(S6) Cooper, R. I.; Thompson, A. L.; Watkin, D. J. J. Appl. Crystallogr., 2010, 43, 1100-1107.

(S7) Cliffe, M. J.; Goodwin, A. L. J. Appl. Crystallogr., 2012, 45, 1321-1329.

(S8) Koch, E.; Fisher, W. Z. Krist., 1978, 148, 107-152.

(S9) Kieslich, G.; Sun, S.; Cheetham, A. K. Chem. Sci., 2014, 5, 4712-4715.

(S10) Shannon, R. D. Acta Crystallogr. Sect. A, 1976, 32, 751-767.

(S11) Cordero, B.; Gomez, V.; Platero-Prats, A. E.; Reves, M.; Echeverria, J.; Cremades, E.; Barragan, F.; Alvarez, S. Dalton Trans., 2008, 2832-2838.

(S12) Kieslich, G.; Kumagai, S.; Butler, K. T.; Okamura, T.; Hendon, C. H.; Sun, S.; Yamashita, M.; Walsh, A.; Cheetham, A. K. Chem. Commun., 2015, 51, 15538-15541.

(S13) Lefebvre, J.; Chartrand, D.; Leznoff, D. B. Polyhedron, 2007, 26, 2189-2199.

(S14) Glazer, A. M. Acta Crystallogr. Sect. B, 1972, 28, 3384-3392.

(S15) Goodwin, A. L.; Kepert, C. J. Phys. Rev. B, 2005, 71.

(S16) Howard, C. J.; Withers, R. L.; Knight, K. S.; Zhang, Z. J. Phys.:Condens. Matter, 2008, 20, 135202. 